\title{
PROBIOTICS EFFECT ON THE GINGIVA OF UNCONTROLLED DIABETIC ALBINO RATS
}

\author{
Samah Mohamed Kamel* and Mohamed Shamel ElKady*
}

\begin{abstract}
Objective: This study was designed to evaluate the effect of using L.Reuteri containing tablets, on the gingiva of uncontrolled diabetic rats both histologically and ultrastructurally.

Material and Methods: Thirty adult male albino rats were equally divided into three groups, Group I served as control, Group II: rats were subjected to induction of diabetes mellitus using streptozocin, Group III: rats treated as group II animals and on the next day they were fed daily a probiotic tablet Prodentis containing L.reuteri for 14 days. At the end of the experiment, histological and ultrastructural examination was performed on the gingiva.
\end{abstract}

Results: Rats of group II revealed inflammatory gingiva with loss of normal structure, while that of group III revealed a somewhat normal gingiva in comparison to group II.

Conclusion: L.reuteri containg tablets can inhibit gingival inflammation associated with diabetics through its antibacterial and autoinflammatory properties.

Keywords: Probiotics, Gingiva, Diabetes

\section{INTRODUCTION}

The oral cavity microbiota is an ecosystem formed nearly by 700 different microbial species. There is a distinctive, predominant bacterial flora of the healthy oral cavity that is highly diverse and site and subject specific. Most sites harbor 20-30 different predominant species, and the number of species varies from 34 to 70 in an individual mouth. ${ }^{(1)}$

Mager et al showed that in healthy subjects, the proportions of 40 oral bacterial species significantly differ on different intraoral surfaces. They also found that the microbiota of the soft tissues were more similar to each other than the microbiota of supragingival and subgingival plaques. (2) This complex system can be altered by diverse factors, including poor hygiene and diet, smoking, stress and systemic diseases. All these perturbations favor the colonization by pathogenic bacteria and the formation of biofilms, which are both etiological factors of gingival and periodontal diseases. ${ }^{(3)}$

* Lecturer Oral Biology, Faculty of Dentistry, October University of Modern Sciences and Art. 
Gingivitis is a bacterial infection-induced inflammation of the gingival tissues surrounding the tooth. Bacterial attachment on the tooth surface and co-adhesion of initial colonizers with other species initiate the formation of a biofilm. Mature biofilm, which is also called bacterial dental plaque, stimulates a cascade of events that leads to gingival inflammation. Gingivitis, either at an acute or chronic state, is a reversible disease, since there is no loss of hard tissues that support the teeth. The gingival tissues return to their healthy state when the bacterial biofilm is removed. ${ }^{(4)}$

Diabetes has been associated with oral infections such as periodontitis, dental caries, gingivitis and candidiasis. ${ }^{(5-7)}$ Several studies have documented the oral microbiota in diabetic patients as well as other patient populations. ${ }^{(8-10)}$

Sharma and coworkers reported that both Gram positive and Gram negative bacteria are fairly involved in dental diseases and that the prevalence of bacteria increases with severity of disease. In another related study, it was reported that periodontal pathogens were different in diabetic patients and non-diabetic controls as well as in aggressive and chronic periodontitis. ${ }^{(2,11)}$

Controlling the colonization of the oral cavity by pathogenic bacteria is essential for the prevention of periodontal diseases. Literature data show that the mechanical removal of supragingival plaque is the most effective tool to prevent gingivitis. However, as individuals often do not handle plaque accumulation, antimicrobial agents, like dentifrices or mouthwashes, have been tested for their additional ability to reduce plaque and gingivitis onset. Another method is represented by the longterm use of antiseptic mouthwashes that, however, may be associated with unwanted side effects. ${ }^{(12)}$

An alternative and preventive tool may be represented by the use of a class of nonpathogenic microorganisms called probiotics, which may increase the commensal flora, preventing the microbiological shift and colonization of pathogens associated with gingival inflammation .(13)

According to World Health Organization (WHO), "probiotics" are defined as "live micro-organisms which, when administered in adequate amounts, confer a health benefit on the host". Probiotics prevent the adhesion of pathogenic species, they inhibit bacterial growth, modulate cell proliferation and the mucosal immune system and improve the integrity of the intestinal barrier. ${ }^{(14)}$

Among which Gram-positive lactic acid bacteria (LAB) are most promising candidates for controlling oral pathogenic bacteria. LAB such as L. reuteri, L. fermentum, and L. casei Shirota have antimicrobial and anti-inflammatory properties. Of these bacteria, L. reuteri is most interesting for the control of oral microbiota as it produces reuterin, a wide spectrum antimicrobial agent. Reuterin inhibits growth of both Gram-positive and Gram-negative bacteria, including oral pathogens S. mutans, Actinomyces actinomycetemcomitans, Prevotella intermedia, Fusobacterium nucleatum, as well as yeasts like Candida albicans, some fungi and protozoa, without affecting the indigenous health-related microbiota. ${ }^{(15-17)}$

Based on the health benefits reported by specific strains or probiotic combination, this study was carried out to evaluate the impact of a new formulation of probiotic oral tablet containing Lactobacilli reuteri (Prodentis) on gingivitis associated with uncontrolled diabetic rats. The hypothesis is that the use of the present probiotic tablet might be effective on the reduction of gingival inflammation in diabetics.

\section{MATERIALS AND METHODS}

Thirty healthy adult male Albino rats, three months old and 200-220 gm body weight were used in this study. The rats were obtained from Kasr el Aini animal experimental unit, Faculty of 
Medicine, Cairo University. The rats were housed in separate cages, five rats per cage and kept in an environment with controlled temperature $\left(25^{\circ} \mathrm{C}\right)$, humidity (45\%-75\%), and photoperiod (12:12 hour light-dark cycle). The animals were fed natural diet and supplied drinking water adlibitum throughout the whole experimental period. The rats were acclimatized for one week before the initiation of the experiment.

The animals were randomly divided into three groups as follows:

Group I: consisted of 10 rats and served as control.

Group II: consisted of 10 rats subjected to induction of diabetes mellitus using streptozocin as follows:

After 16 hours of fasting, streptozocin (STZ) was injected intraperitoneally as a single dose of $60 \mathrm{mg} /$ $\mathrm{Kg}$ body weight, after being freshly prepared by dissolving $100 \mathrm{mg} \mathrm{STZ} / 20 \mathrm{ml}$ of cold $0.1 \mathrm{M}$ sodium citrate buffer. ${ }^{(18)}$

Group III: Consisted of 10 rats treated as group II animals. On the next day they were fed daily a probiotic tablet Prodentis containing Lactobacilli reuteri dissolved in saline through an oral tube for 14 days.

At the end of the experiments, the animals of the whole groups were sacrificed by cervical dislocation and the samples of the gingiva of the four quadrants were be dissected out.

Those of the right side were fixed in $10 \%$ neutral buffered formalin, processed as usual and embedded in paraffin, then sectioned and used for Hematoxylin and eosin stain for structural examination.

The specimens of the left sides will be used for Ultrastructural examination, where small specimens one cubic mm will be cut, fixed in 3\% glutaraldehyde and prepared for transmission electron microscopic examination.

\section{RESULTS}

\section{Group I:}

\section{Histological results:}

The gingiva of the control animals showed the normal histological features of the surface epithelium and lamina propria. The surface epithelium was of the keratinized stratified squamous type characterized by numerous foldings towards the underlying connective tissue of the lamina propria forming numerous slender, long and irregular epithelial ridges. (Fig.1)

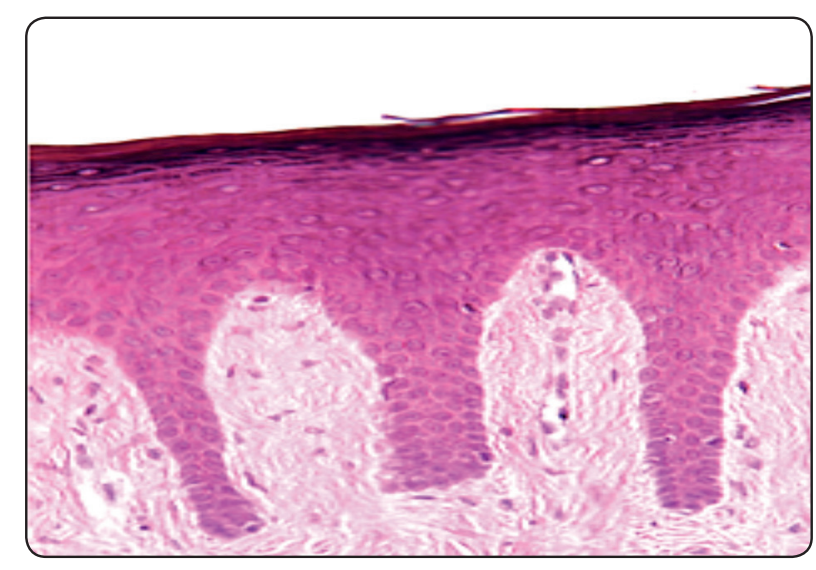

Fig. (1) A photomicrograph of the gingiva of the control group showing the surface epithilium with irregular epithilial ridges (H\&EX200). 


\section{Ultrastructural results:}

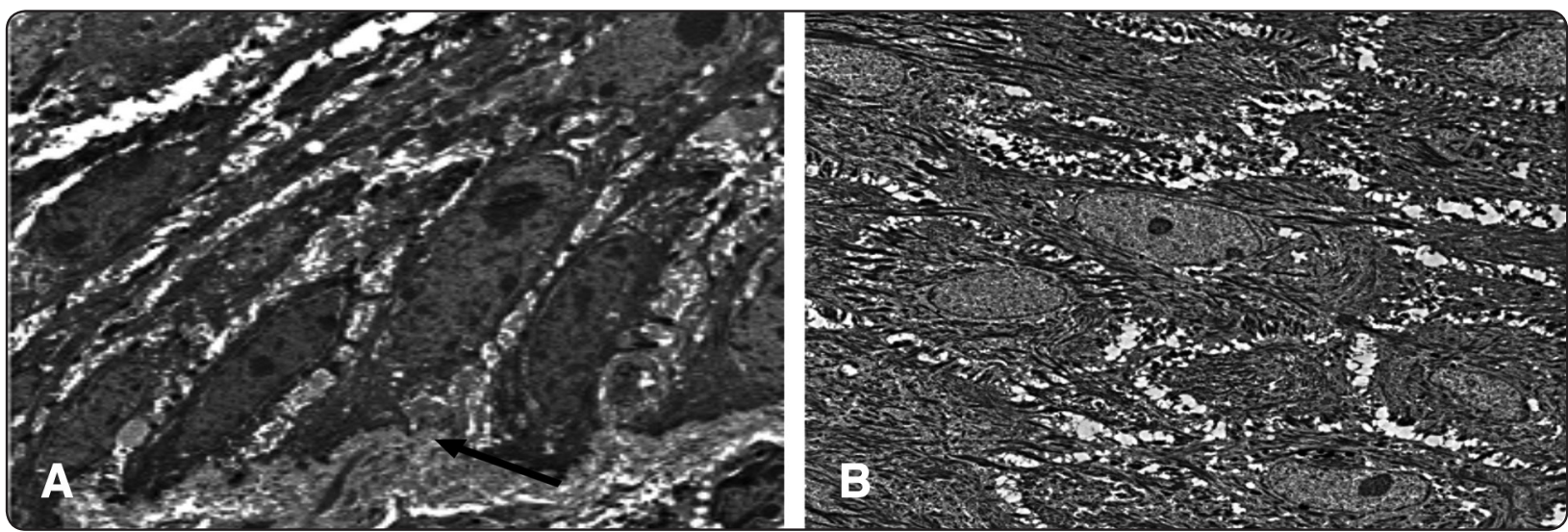

Fig 2: Electron micrograph showing a) normal basal lamina (black arrow), b) prickle cells with normal nuclei and the cells are connected with desmosomes

\section{Group II:}

\section{Histological results:}

Specimens of the gingiva of animals that received a single dose of streptozocin different histological features than that of the control rats where there was an increase in the thickness of the keratin layer and slight intracytoplasmic vacuolization of some of the epithelial cells. The lamina propria demonstrated dissociation in the collagen fibers with infiltration of inflammatory cells and dilatation of the blood vessels (Fig. 3).

\section{Ultrastructural results:}

Ultrastructural examination of gingiva specimens of group II revealed irregular basal lamina, basal cells with pyknotic nuclei, prickle cells deformed nuclei with condensed and peripheralized chromatin with wide intercellular space in some places. (Fig. 4)

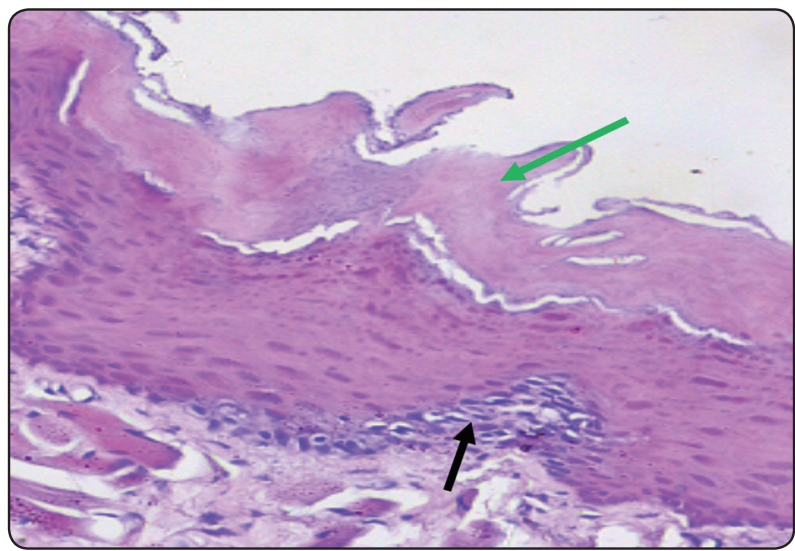

Fig. (3) A photomicrograph of the gingiva of group II showing inflamed gingiva with hyperkeratosis (green arrow) and sub epithelial inflammatory cells (black arrow) (H \& E X400).
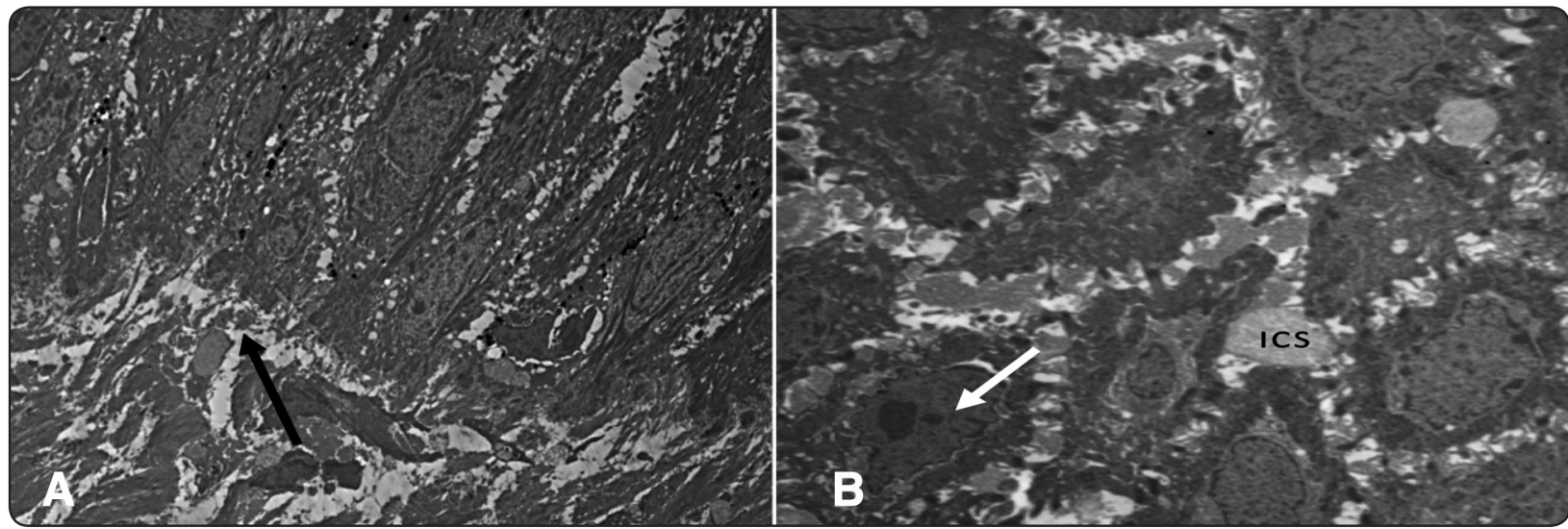

Fig. (4) Electron micrograph showing a) irregular basal lamina (black arrow), b) prickle cells with deformed nuclei with condensed and peripheralized chromatin (white arrow) with wide intercellular space (ICS) in some places 


\section{Group III:}

\section{Histological results:}

Specimens of the gingiva of animals which received a single dose of streptozocin followed by daily probiotic tablet containing kaza kaza for 14 days showed nearly similar histological features of the control rats with normal keratin thickness, slightly wide epithelial ridges and remarkable mitotic figures in the basal cells. The lamina propria showed its normal structure with absence of inflammatory cells (Figure 5).

\section{Ultrastructural Results:}

Ultrastructural examination of gingiva specimens of group III showed architecture similar to that of control group. Regular basal lamina was found with normal nuclei present in the basal cells with mitotic figures also present. Prickle cells showed narrower intercellular spaces in comparison to that of group II.

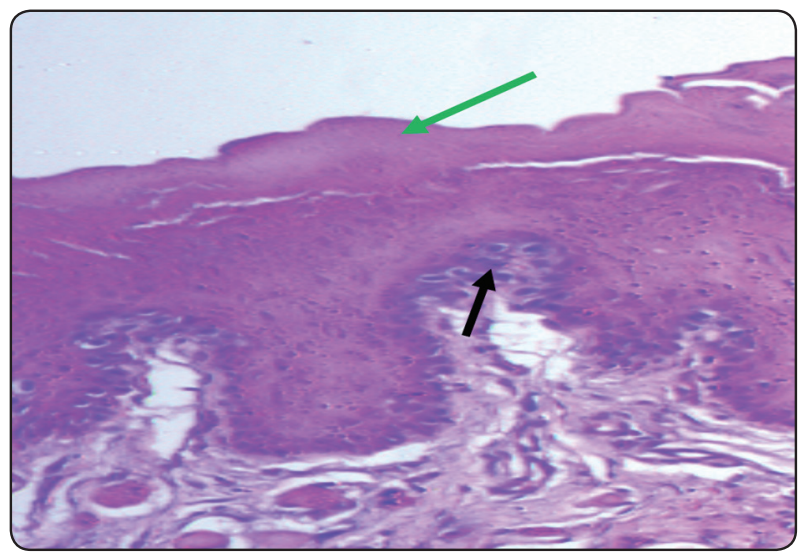

Fig (5) A photomicrograph of the gingiva of group III showing normal keratin layer (green arrow) and the basal cells shows remarkable mitotic figures (black arrow) (H \& E X400).
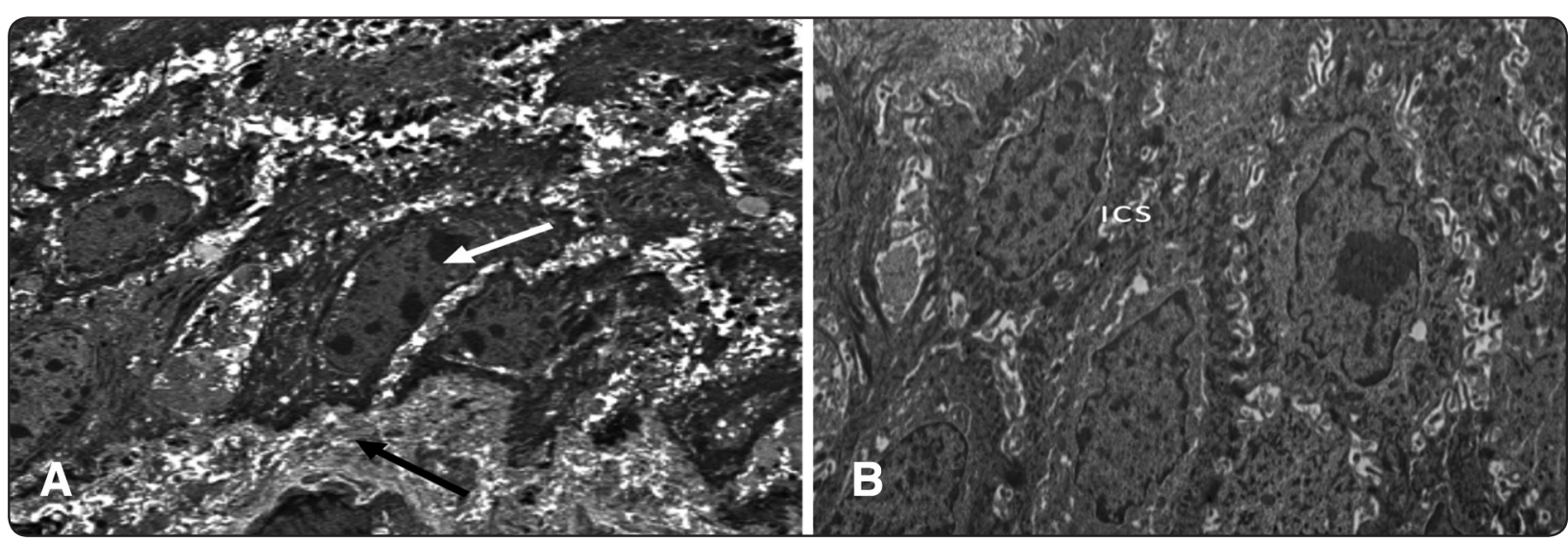

Fig. (6) Electron micrograph showing a) regular basal lamina ( black arrow), normal nuclei with mitotic figure (white arrow), b) prickle cells with less intercellular space (ICS)

\section{DISCUSSION}

The results of this investigation clearly demonstrate a significant major association between the regular consumption of L. reuteri-containing oral tablets and the reduction in gingival inflammation associated with uncontrolled diabetic rats.

In this study, probiotic containing Lactobacillus Reuteri was used for its known antimicrobial properties against pathogenic bacteria.
The results of the current study revealed that uncontrolled diabetic rats suffered from severe gingivitis in comparison to the control group which showed no inflammatory status.

These findings are consistent with many studies that showed that gingival and periodontal inflammation are major complications associated with diabetics especially uncontrolled diabetes. ${ }^{(19)}$

This is due to elevated levels of systemic markers of inflammation associated with diabetes 
mellitus type 1 and 2 The elevated inflammatory state in diabetes contributes to both microvascular and macrovascular complications, and it is clear that hyperglycaemia can result in the activation of pathways that increase inflammation, oxidative stress and apoptosis. Thus it can be concluded from the previous data, that diabetes is directly associated with increased levels of gingivitis and periodontitis. ${ }^{(20,21)}$

Another explanation to the increased susceptibility to periodontitis associated with diabetes is apoptosis in which apoptosis of fibroblasts may limit the opportunities for repair in inflamed tissues. Induction of tissue injury by inoculation with $P$. gingivalis resulted in significantly higher fibroblast apoptosis in diabetic mice compared with non-diabetic mice, indicating another mechanism by which diabetes can interfere with the capacity for repair in inflamed periodontal tissues. Moreover, many kinds of bacteria thrive on sugars, including glucose the sugar linked to diabetes. When diabetes is poorly controlled, high glucose levels in mouth fluids may help germs grow and set the stage for gingival and periodontal disease. ${ }^{(22,23)}$

In contrary, the histological study of the gingival tissue of the third group which received daily dosage of L.Reuteri containing tablets, showed no to mild gingival inflammation.

They confirm the clinical findings of Twetman et al. reporting a significant beneficial influence of L. reuteri-containing chewing gums on the manifestation of bleeding on probing in a cohort of young adults suffering from chronic gingivitis. ${ }^{(24)}$

The results of the current study also confirms the study performed by Krasse et al which carried out a double-blind, placebo-controlled study to investigate the effects of a $\mathrm{L}$. reuteri probiotic lozenge on 59 volunteers with moderate to severe gingivitis. Following 14 days of probiotic treatment, a highly significant decrease in inflammation was observed and plaque accumulation scores also decreased. ${ }^{(25)}$
The improvement in the gingival condition can be explained by L. reuteri which reportedly produces a number of antibacterial compounds including, reuterin, a broad-spectrum antimicrobial structurally related to hydroxypropionaldehyde, along with the fermentation products lactic and acetic acid. The inhibitory effect of reuterin has been claimed to select against pathogenic oral bacteria and it seems likely that lactobacilli will not be highly sensitive to this compound, given its origin. Accordingly, L. reuteri has inhibited potentially harmful species in vitro and in human volunteer studies, including the cariogenic bacterium S. mutans and various periodontal pathogens.

Moreover, the improved gingival condition can be related to the significant reduction in cytokines TNF- $\alpha$ and IL $1 \beta$ which was earlier confirmed by a study performed by Twetman et al which examined short term effect of chewing gums containing probiotic Lactobacillus reuteri on the levels of inflammatory mediators in gingival crevicular fluid. The authors reported significant reduction in Cytokines TNF- $\alpha$ and IL1 $\beta$, which are considered central mediators of proinflammatory cascade causing damage. ${ }^{(14)}$

Another explanation for the reduced gingivitis in the diabetic rats receiving L.Reuteri is the inhibitory effect of L.Reuteri on P.Gingivalis. P. gingivalis is an anaerobic, Gram-negative, rod-shaped and highly virulent organism implicated as a major pathogen in gingival and destructive periodontal disease with its ability to adhere and invade oral epithelium As mentioned before, P.Gingivalis is associated with higher rates of fibroblast apoptosis in diabetics so inhibiting its presence by L.Reuteri causes a decrease in apoptosis thus fibroblasts have a higher ability to repair inflamed gingival and periodontal tissues, in addition to decreasing the direct pathogenic effect of P.Gingivalis on the periodontal tissues. 


\section{REFERENCES}

1. Aas JA, Paster B J, Stokes L N, Olsen I,Dewhirst, FE. Defining the normal bacterial flora of the oral cavity. J Clin Microbiol; 43(11): 5721-5732,2005.

2. Mager DL, Ximenez-Fyvie LA, Haffajee AD, Socransky SS .Distribution of selected bacterial species on intraoral surfaces. J Clin Periodontol; 30(7):644-654,2003.

3. Nazir MA. Prevalence of periodontal disease, its association with systemic diseases and prevention. Int J Health Sci (Qassim); 11(2): 72-80,2017.

4. Filoche S, Wong L, Sissons CH .Oral biofilms: emerging concepts in microbial ecology. J Dent Res; 89(1): 8-18,2010.

5. Preshaw PM, Alba AL, Herrera D, Jepsen S, Konstantinidis A, Makrilakis K, Taylor R. Periodontitis and diabetes: a two-way relationship. Diabetologia; 55(1): 21-31,2012.

6. Singh I, Singh P, Singh A, Singh T, Kour R .Diabetes an inducing factor for dental caries: A case control analysis in Jammu. J Int Soc Prev Community Dent; 6(2):125-129,2016.

7. Casqueiro J , Casqueiro J, Alves C. Infections in patients with diabetes mellitus: A review of pathogenesis. Indian $\mathrm{J}$ Endocrinol Metab;16(Suppl1): S27-36,2012.

8. Anbalagan R, Srikanth P, Mani M, Barani R, Seshadri KG, Janarthanan R. Next generation sequencing of oral microbiota in Type 2 diabetes mellitus prior to and after neem stick usage and correlation with serum monocyte chemoattractant-1. Diabetes Res Clin Pract; 130: 204-210,2017.

9. Ogawa T, Honda-Ogawa M, Ikebe K, Notomi Y, Iwamoto Y, Shirobayashi I, Maeda Y .Characterizations of oral microbiota in elderly nursing home residents with diabetes. J Oral Sci;59(4): 549-555,2017.

10. Ohlrich EJ, Cullinan MP, Leichter JW. Diabetes, periodontitis, and the subgingival microbiota. J Oral Microbiol; 2,2010.

11. Sharma M, Tiwari SC, Singh K, Kishor K.Occurrence of bacterial flora in oral infections of diabetic and nondiabetic patients. Life Sci. Med. Res.; 32, 2011.

12. Ercan N, Erdemir EO, Ozkan SY, Hendek MK. The comparative effect of propolis in two different vehicles; mouthwash and chewing-gum on plaque accumulation and gingival inflammation. Eur J Dent; 9(2): 272-276,2015.

13. Kumar VN, Krishnamurthy M, Poorni S, Patil S, Raj AT. Probiotics in Caries Prevention. J Contemp Dent Pract;19(2):123-124,2018.

14. Twetman S, Keller MK. Probiotics for caries prevention and control. Adv Dent Res; 24(2): 98-102,2012.
15. Brown AC, Valiere A. Probiotics and Medical Nutrition Therapy. Nutr Clin Care; 7(2): 56-68,2004.

16. Sanchez-Maldonado AF, Schieber A, Ganzle MG. Structure-function relationships of the antibacterial activity of phenolic acids and their metabolism by lactic acid bacteria. J Appl Microbiol; 111(5): 1176-1184,2011 .

17. Baca-Castanon ML, De la Garza-Ramos MA, AlcazarPizana AG, Grondin Y, Coronado-Mendoza A, SanchezNajera RI, Escamilla-Garcia E. Antimicrobial Effect of Lactobacillus reuteri on Cariogenic Bacteria Streptococcus gordonii, Streptococcus mutans, and Periodontal Diseases Actinomyces naeslundii and Tannerella forsythia. Probiotics Antimicrob Proteins; 7(1): 1-8,2015.

18. Fahim MA,Hasan MY,Alshuaib WB. Early morphological remodeling of neuromuscular junction in a murine model of diabetes. J Appl Physiol ;89(6):2235-40,2000.

19. Chapple IL, Genco R. Diabetes and periodontal diseases: consensus report of the Joint EFP/AAP Workshop on Periodontitis and Systemic Diseases. J Periodontol;84(4 Suppl):S106-112,2013.

20. Mesia R, Gholami F, Huang H, Clare-Salzler M, Aukhil I, Wallet SM, Shaddox LM. Systemic inflammatory responses in patients with type 2 diabetes with chronic periodontitis. BMJ Open Diabetes Res Care;4(1),2016.

21. Chawla A, Chawla R, Jaggi S. Microvasular and macrovascular complications in diabetes mellitus: Distinct or continuum? Indian J Endocrinol Metab;20(4):546-551,2016.

22. D'Aiuto F, Parkar M, Andreou G, Suvan J, Brett PM, Ready D, Tonetti MS. Periodontitis and systemic inflammation: control of the local infection is associated with a reduction in serum inflammatory markers. J Dent Res; 83(2): 156-160,2004.

23. Marcaccini AM, Meschiari CA, Sorgi CA, Saraiva MC, de Souza AM, Faccioli LH, Gerlach RF. Circulating interleukin-6 and high-sensitivity C-reactive protein decrease after periodontal therapy in otherwise healthy subjects. J Periodontol; 80(4):594-602,2009.

24. Twetman S, Derawi B, Keller M, Ekstrand K, Yucel-Lindberg T, Stecksen-Blicks C. Short-term effect of chewing gums containing probiotic Lactobacillus reuteri on the levels of inflammatory mediators in gingival crevicular fluid. Acta Odontol Scand; 67(1):19-24,2009.

25. Krasse P, Carlsson B, Dahl C, Paulsson A, Nilsson A, Sinkiewicz G . Decreased gum bleeding and reduced gingivitis by the probiotic Lactobacillus reuteri. Swed Dent J; 30(2):55-60,2006. 